\title{
AUTOMATIC CROSSOVER FREQUENCY SELECTION FOR MULTICHANNEL HOME-THEATER APPLICATIONS
}

\author{
Sunil Bharitkar, Chris Kyriakakis \\ Audyssey Labs., Inc., and \\ University of Southern California, \\ Los Angeles, USA
}

\begin{abstract}
Given a multichannel loudspeaker system, the selection of the crossover frequency between the sub-woofer and the satellite speakers is important for accurate (i.e., distortionfree), reproduction of playback sound. Presently, many hometheater systems have selectable crossover frequencies which are either set by the consumer through listening tests. Alternatively, if the loudspeakers are industry certified, the crossover frequency is set at $80 \mathrm{~Hz}$. A desirable feature is that, besides distortion-free sound output from the individual subwoofer and the satellite speakers, the combined sub-woofer and satellite room acoustical response should exhibit negligible variations around the selected crossover frequency. In this paper, we present an automatic crossover frequency selection algorithm based on an objective measure (viz., the spectral deviation measure) for multichannel home-theater applications that allows better control of the combined subwoofer-satellite response, thereby significantly improving audio quality.
\end{abstract}

\section{INTRODUCTION}

A typical room is an acoustic enclosure that can be modelled as a linear system whose behavior at a particular listening position is characterized by an impulse response, $h(n) ; n \in$ $\{0,1,2, \ldots\}$ with an associated frequency response or room transfer function $H\left(e^{j \omega}\right)$. The impulse response yields a complete description of the changes a sound signal undergoes when it travels from a source to a receiver (microphone/listener). The signal at a listening position consists of direct path components, discrete reflections that arrive a few milliseconds after the direct sound, as well as a reverberant field component.

A typical 5.1 a system level description is shown in Fig. 1, where the satellites (left, center, right, left surround and right surround speakers) are positioned surrounding the listener and the sub-woofer may be placed in the corner or near the edges of a wall. The high-pass (satellite) and lowpass (subwoofer) bass management filters, used in the au- dio industry, $\left|H_{b m, \omega_{c}}^{h p}(\omega)\right|=1-1 / \sqrt{1+\left(\omega / \omega_{c}\right)^{4}}$ and $\left|H_{b m, \omega_{c}}^{l p}(\omega)\right|=1 / \sqrt{1+\left(\omega / \omega_{c}\right)^{8}}$, are Butterworth second order high-pass (12 dB/octave roll-off) and fourth order lowpass ( $24 \mathrm{~dB} /$ octave roll-off), respectively, and are designed with a crossover frequency $\omega_{c}$ (i.e., the intersection of the corresponding $3 \mathrm{~dB}$ points) corresponding to $80 \mathrm{~Hz}$. Examples of other crossover networks that split the signal energy between the subwoofer and the satellites, according to predetermined crossover frequency and slopes, can be found in $[1,2,3]$. The magnitude responses of the individual bass management filters, used in industry, as well as the magnitude of the recombined response (i.e., the magnitude of the complex sum of the filter frequency responses), are shown in Fig. 2. Thus, the selection of the crossover frequency is an extremely important problem and the selection should be such that the subwoofer and the satellite channel output is distortion-free.

The acoustical block diagram for a subwoofer channel and a satellite channel is shown in Fig. 3, where $H_{s u b}(\omega)$ and $H_{\text {sat }}(\omega)$ are the acoustical loudspeaker-room responses at a listening position. The resulting net acoustic transfer function, $H(\omega)$, and magnitude response, $\left|H\left(e^{j \omega}\right)\right|^{2}$ can be written as

$$
\begin{aligned}
H(\omega) & =H_{b m, \omega_{c}}^{h p}(\omega) H_{\text {sat }}(\omega) \\
+ & H_{b m, \omega_{c}}^{l p}(\omega) H_{s u b}(\omega) \\
|H(\omega)|^{2}= & |A(\omega)|^{2}+|B(\omega)|^{2}+\Gamma(\omega) \\
|A(\omega)|^{2}= & \left|H_{b m, f_{c}}^{h p}(\omega)\right|^{2}\left|H_{s a t}(j \omega)\right|^{2} \\
|B(\omega)|^{2}= & \left|H_{b m, f_{c}}^{l p}(\omega)\right|^{2}\left|H_{s u b}(\omega)\right|^{2} \\
\Gamma(\omega)= & 2|A||B| \cos \left(\phi_{s u b}(\omega)+\phi_{b m, \omega_{c}}^{l p}(\omega)\right. \\
& \left.-\phi_{\text {sat }}(\omega)-\phi_{b m, \omega_{c}}^{h p}(\omega)\right)
\end{aligned}
$$

where $\phi_{b m, \omega_{c}}^{h p}(\omega)$ and $\phi_{b m, \omega_{c}}^{l p}(\omega)$ are the phase responses of the bass management filters, whereas $\phi_{s u b}(\omega)$ and $\phi_{\text {sat }}(\omega)$ are the phase responses of the subwoofer-room and satelliteroom responses.

However, many of the loudspeaker systems, in a real 
room, interact with the room giving rise to standing wave phenomena which manifests are significant variations in the magnitude response measured between a loudspeaker and a microphone position. As can be readily observed from (1), with an incorrect crossover frequency choice, the phase interactions will show up in the magnitude response as a region with a broad spectral notch indicating in a substantial attenuation of sound around the crossover region. In this paper, we show that a correct choice of the crossover frequency will influence the combined magnitude response around the crossover region.

As an example, individual sub-woofer and satellite (in this case a center channel) frequency responses (1/3-rd octave smoothed), as measured in a room at a sampling frequency of $48 \mathrm{kHz}$ with a reverberation time $T_{60} \approx .75$ sec., are shown in Figs. 4(a) and 4(b) respectively. Clearly, the satellite is capable of playing audio below $100 \mathrm{~Hz}$ (up to about $40 \mathrm{~Hz}$ ), whereas the sub-woofer is most efficient and generally used for audio playback at frequencies less than $200 \mathrm{~Hz}$. For example, as shown in Fig. 5, the resulting magnitude response, according to (1), obtained by summing the impulse responses, has a severe spectral notch for a crossover frequency $\omega_{c}$ corresponding to $60 \mathrm{~Hz}$. This has been verified through real measurements where the subwoofer and the satellite channels were excited with a broadband stimuli (e.g., log-chirp signal) and subsequently deconvolving the net response from the measured signal.

While room equalization has been widely used to solve problems in the magnitude response, the equalization filters do not necessarily solve the problems around the crossover frequency. In fact, many of these filters are minimum phase and as such may do little to influence the result around the crossover. However, while the technique described in this paper is applied primarily to responses before equalization, it can be readily applied after equalization to the equalization filters.

Thus, in this paper, we present an approach for minimizing the variations due to phase interaction between noncoincident speakers for better magnitude response control around the crossover. The analysis and results presented in this paper does not include the situation where an equalization filter is present in each channel, however it is easy to extend the principles of this paper to this situation so as to achieve a substantially flat magnitude response in the crossover region. Accordingly, section 2 presents an objective function based scheme, for selecting a crossover frequency, for minimizing the spectral notch in the magnitude response around the crossover. Results obtained from using this technique will also be presented along. Section 3 presents some interpretation of the results demonstrating the influence of the phase interaction terms $\Gamma(\omega)$ around the crossover region, while section 4 concludes the paper and presents future directions.

\section{OBJECTIVE FUNCTION BASED CROSSOVER FREQUENCY SELECTION}

An objective function that is particularly useful for characterizing the magnitude response is the spectral deviation measure [5], [6]. Given that the effects of the choice of the crossover frequency are bandlimited around the crossover frequency, it will be shown that this measure is quite effective in predicting the behavior of the resulting magnitude response around the crossover. The spectral deviation measure, $\sigma_{H}\left(\omega_{c}\right)$, which indicates the degree of flatness of the magnitude spectrum is defined as,

$$
\sigma_{H}\left(\omega_{c}\right)=\sqrt{\left[\frac{1}{P} \sum_{i=0}^{P-1}\left(10 \log _{10}\left|H\left(\omega_{i}\right)\right|-\Delta\right)^{2}\right]}
$$

where $\Delta=1 / P \sum_{i=0}^{P-1} 10 \log _{10}\left|H\left(\omega_{i}\right)\right|,\left|H\left(\omega_{i}\right)\right|$ can be found from (1), and $P$ is the number of frequency points selected around the crossover region. Specifically, the smaller the $\sigma_{H}\left(\omega_{c}\right)$ value, the flatter is the magnitude response.

For real-time applications, a typical home theater receiver includes a selectable (either by an user or automatically as shown by this paper) finite integer set of crossover frequencies. For example, typical home-theater receivers has selectable crossover frequencies, in $10 \mathrm{~Hz}$ increments, from $20 \mathrm{~Hz}$ through $150 \mathrm{~Hz}$ (i.e., $\Omega=[20 \mathrm{~Hz}, 30 \mathrm{~Hz}, 40$ Hz.,..., $150 \mathrm{~Hz}]$ ). Thus, although a near-optimal solution $\omega_{c}^{*}$ can be found through a gradient descent optimization process by minimizing the spectral deviation measure with respect to $\omega$ (viz., $\partial \sigma_{H}\left(\omega_{c}\right) /\left.\partial \omega_{c}\right|_{\omega_{c}=\omega_{c}^{*}}$ ), this is unnecessarily complicated. Clearly, the choice of the crossover frequency is limited to this finite set of integers (viz., as given in $\Omega$ ), hence a simpler but yet effective means, to select a proper choice of the crossover frequency, is to characterize the effect of the choice of each of the selectable integer crossover frequency on the magnitude response in the crossover region.

Fig. 6 show the resulting magnitude responses, as obtained via (1), for different integer choices of the crossover frequencies from $50 \mathrm{~Hz}$ through $130 \mathrm{~Hz}$. The corresponding spectral deviation values, as a function of the crossover frequency, for the crossover region around the crossover frequencies are shown in Fig. 7. Clearly, comparing Fig. 7 results with the plots in Figs. 6, it can be clearly seen that the spectral deviation measure is an excellent measure for accurately modelling the performance in the crossover region for a given choice of crossover frequency. The best crossover frequency is then that which minimizes the spectral deviation measure, in the crossover region, over the integer set of crossover frequencies. Specifically,

$$
\omega_{c}^{*}=\min _{\omega_{c} \in \Omega} \sigma_{H}\left(\omega_{c}\right)
$$


In this example $120 \mathrm{~Hz}$ provided the best choice for the crossover frequency as it gave the smallest $\sigma_{H}\left(\omega_{c}\right)$.

\section{THE INFLUENCE OF PHASE ON THE NET MAGNITUDE RESPONSE}

In this section, we briefly explain some of the results obtained in the previous section. Specifically, we demonstrate that an appropriate crossover frequency enables coherent addition of the phase interaction term $\Gamma(\omega)$ with the $|A(\omega)|^{2}$ and $|B(\omega)|^{2}$ terms in (1).

For example, Fig. 8(b) shows the $\Gamma(\omega)$ term for crossover frequency $\omega_{c}$ corresponding to $60 \mathrm{~Hz}$. Clearly, this term is negative and will contribute to an incoherent addition in (1) around the crossover region (marked by arrows). In contrast, by selecting the crossover frequency to be $100 \mathrm{~Hz}$, the $\Gamma(\omega)$, as shown in Fig. 8(a), is positive around the crossover region. This results in a coherent addition of around the crossover region. These complex addition results are clearly reflected in the plots of Figs. 6(a) and (f) as well as the $\sigma_{H}\left(\omega_{c}\right)$ values at $60 \mathrm{~Hz}$ and $100 \mathrm{~Hz}$ in Fig. 7.

\section{CONCLUSIONS AND FUTURE DIRECTIONS}

In this paper, we presented an automatic crossover frequency selection algorithm for selecting the best possible crossover frequency, from a finite integer set of potential crossover frequencies, using the spectral deviation criteria. The advantages include a computationally simple but yet effective method for selecting the best crossover frequency for optimizing the magnitude response around the crossover region. Future directions will be directed towards selecting a crossover frequency and optimizing the net response for multiple-listener (viz., multiple-response) applications.

\section{ACKNOWLEDGEMENT}

The authors would like to thank Prof. Tom Holman (USC) for discussions on this topic.

\section{REFERENCES}

[1] W. Woszczyk, "Bessel Filters as Loudspeaker Crossovers," Proc. of 72nd AES Conv., 1982,(Preprint 1949).

[2] S. Lipshitz and J. Vanderkooy, "A Family of Linear-Phase Crossover Networks of High Slope Derived by Time Delay," Proc. of 69th AES Conv., 1981, (Preprint 1801).

[3] N. Thiele, "Loudspeaker Crossovers with Notched Responses ," Proc. of 108th AES Conv., 2001, (Preprint 5106).

[4] S. Bharitkar and C. Kyriakakis, "A Cluster Centroid Method for Room Response Equalization at Multiple Locations,"
Proc. of 2001 IEEE Workshop on Applications of Signal Processing to Audio and Acoustics (WASPAA 2001). New Paltz, NY. Oct. 2001.

[5] S. Bharitkar and C. Kyriakakis, "A Comparison Between Multi-channel Audio Equalization Filters Using Warping," Proc. 2003 IEEE Workshop on Applications of Signal Processing to Audio and Acoustics (WASPAA 2003). New Paltz, NY. Oct. 2003.

[6] B. Radlović and R. Kennedy, "Nonminimum-phase Equalization and its Subjective Importance in Room Acoustics," IEEE Trans. on Speech and Audio Proc., vol. 8(6), pp. 728-737, Nov. 2000.

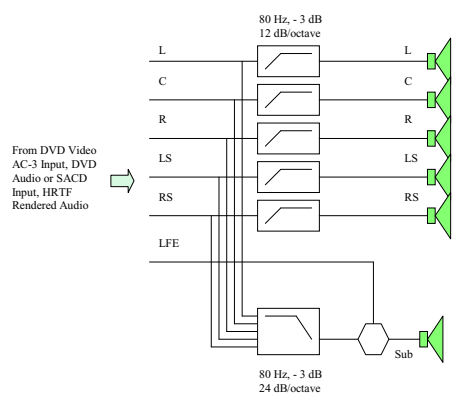

Fig. 1. System level description of the 5.1 multichannel system of Fig. 1.

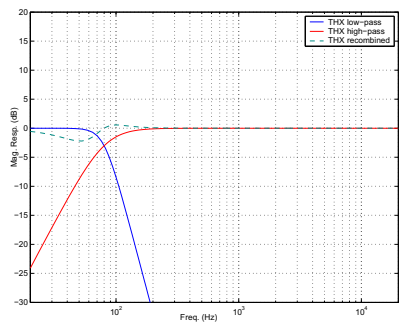

Fig. 2. Magnitude response of the industry standard bass management filters and the recombined response.

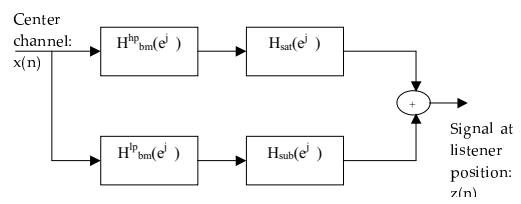

Fig. 3. Block diagram for the combined acoustical response at a position. 

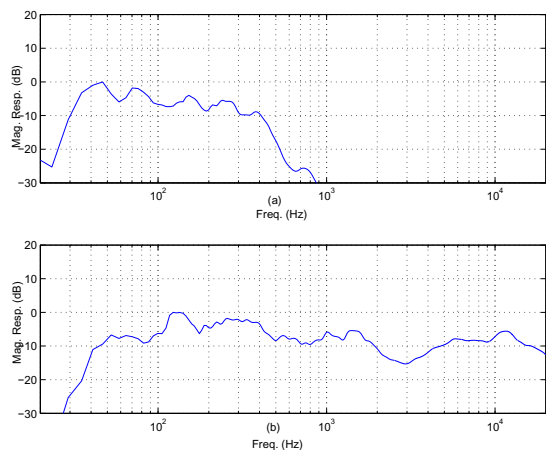

Fig. 4. (a) Magnitude Response of the sub-woofer measured in a reverberant room, (b) magnitude response of the satellite measured in the same room.

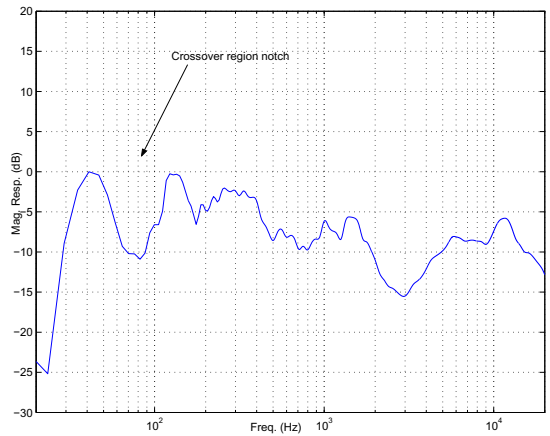

Fig. 5. Magnitude of the net response obtained from using a crossover frequency of $60 \mathrm{~Hz}$.
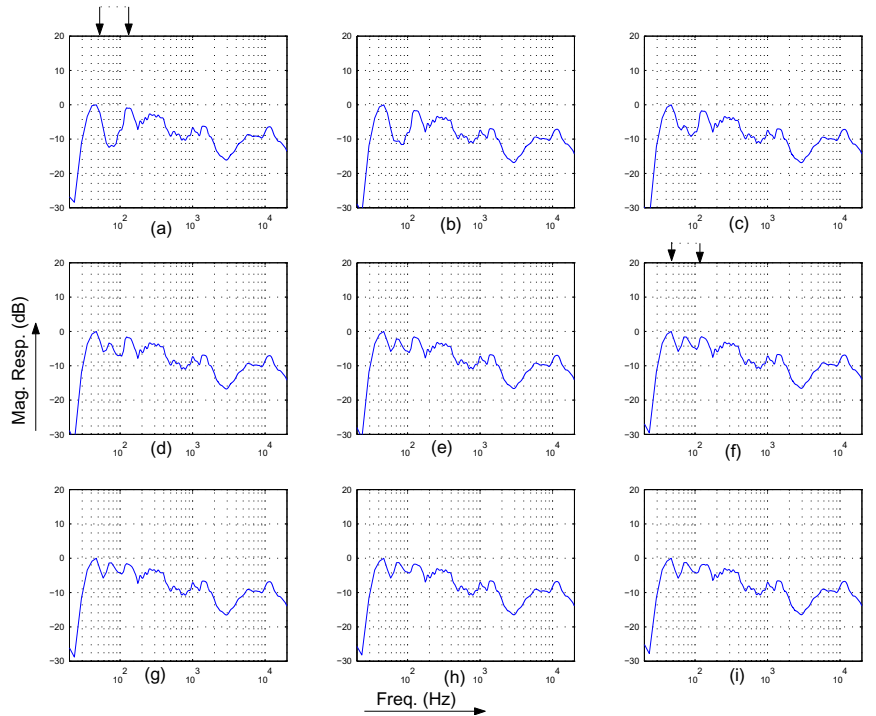

Fig. 6. Plots of the resulting magnitude response for crossover frequencies :(a) $50 \mathrm{~Hz}$, (b) $60 \mathrm{~Hz}$, (c) $70 \mathrm{~Hz}$, (d) $80 \mathrm{~Hz}$, (e) $90 \mathrm{~Hz}$, (f) $100 \mathrm{~Hz}$, (g) $110 \mathrm{~Hz}$, (h) $120 \mathrm{~Hz}$, (i) 130

$\mathrm{Hz}$.

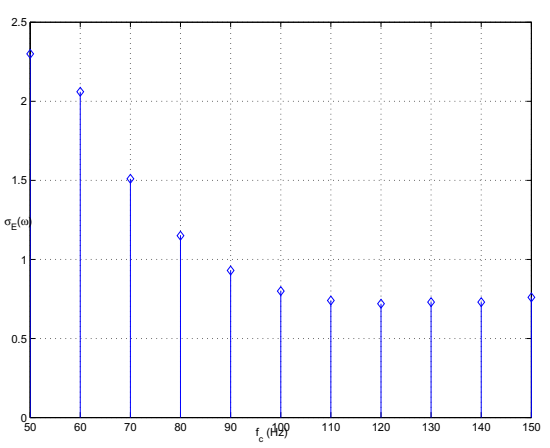

Fig. 7. Spectral deviation versus crossover frequency.
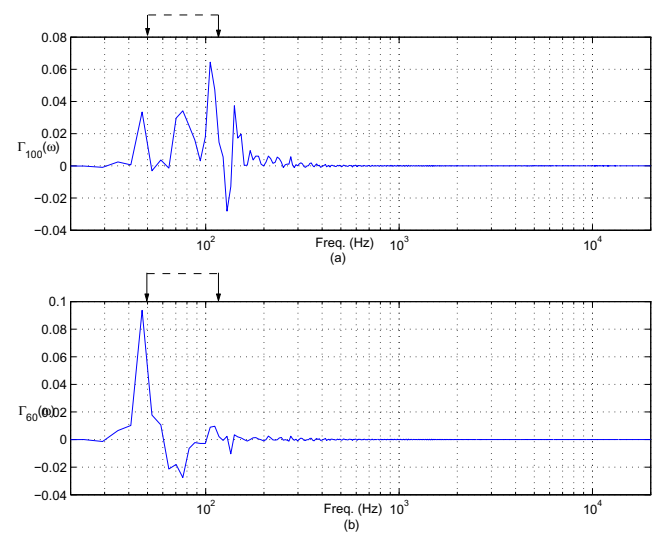

Fig. 8. $\Gamma(\omega)$ term from (1) for crossover frequency (a) 100 $\mathrm{Hz}$, (b) $60 \mathrm{~Hz}$. 